\title{
O SISTEMA DE SAÚDE BRITÂNICO APÓS AS REFORMAS DE 1991: UMA AVALIAÇÃO INICIAL
}

\begin{abstract}
MARCO AKERMAN(*)
O Sistema de Saúde Britânico (NHS) sofreu a mais radical mudança após 1948, quando iniciou suas atividades. Este artigo apresenta uma avaliação inicial das reformas efetuadas, procurando mostrar o atual debate travado na sociedade britânica. Em primeiro lugar discutem-se as estatísticas apresentadas pelo governo como prova do sucesso das reformas. Em seguida, identifica-se o debate atual em relação aos aspectos mais radicais da reforma, a separação entre o ato de comprar serviços e a ação de prover serviços (purchaser) provider split), e o repasse de recursos financeiros para que sejam administrados diretamente por algumas clínicas de general practitioners - GPs. Apresenta ainda o recente plano de governo (Tomlinson Report), que propos o fechamento e fusāo de alguns hospitais de ensino na cidade de Londres. Ao final do artigo aponta depoimentos que caracterizariam o debate atual em relação às reformas: fim do welfare state ou modelo de gestão para o próximo século?
\end{abstract}

\section{INTRODUÇÃO}

Em abril de 1991 o Sistema de Saúde Britânico (NHS) sofreu a sua mais radical mudança após 1948, ano em que iniciou as suas atividades como sistema universalizado financiado pelo tesouro público. Os principais aspectos desta mudança estão descritos e comentados em vários artigos e documentos oficiais ${ }^{1,2,3}$.

(*) Pesquisador associado da London School of Hygiene and Tropical Medicine / Fundação SEADE e PhD em avaliação de senviços de saúde pela Universidade de Londres 
Desde então o NHS vem passando por intenso escrutínio público manifestado pela presença quase diária de reportagens nos jornais, rádios e televisões britânicos, bem como em publicações científicas. Até abril de 1992, data da última eleição para o Parlamento britânico, as reformas ainda não haviam adquirido caráter nacional amplo. Isto porque, o Partido Trabalhista (Labour), propalava que caso vencesse as eleições teria como primeiro ato de governo a anulação das reformas propostas pelo Partido Conservador.

Entretanto, como o resultado das eleições mostrou a vitória dos conservadores, as reformas não estāo mais ameaçadas e parece que chegaram para ficar. A tendência agora é que elas se expandam para todo o Reino Unido e se configurem na primeira experiência mundial de implantação de um mercado interno dentro de um setor público de saúde.

Este artigo faz em primeiro lugar uma análise geral das estatísticas apresentadas pelo governo como prova do sucesso das reformas. Em seguida, identifica o debate atual em relação aos aspectos mais radicais da reforma, a separação entre o ato de comprar serviços e a ação de prover serviços (purchaser/provider split), e o repasse de recursos financeiros para que sejam administrados diretamente por algumas clínicas de general practitioners - GPs. Finalmente, relata e discute o recente plano de governo (Tomlinson Report) ${ }^{4}$, que propôs o fechamento e fusão de alguns hospitais de ensino na cidade de Londres. 


\section{O DEBATE DAS ESTATÍSTICAS GOVERNAMENTAIS}

Em janeiro de 1992 o Departamento de Saúde britânico lançou um relatório onde apresentou uma avaliação preliminar dos resultados obtidos pela implementação das reformas até aquela data ${ }^{5}$. Como comentário geral e introdutório o governo declarou que "as mudanças levaram a uma melhoria da qualidade dos serviços, maior rapidez na resolução de problemas individuais, e melhor utilização de recursos financeiros".

Este relatório, comparando os anos fiscais de 1991-92 com 1990-91, prossegue com a sua declaração de sucesso apresentando estatísticas referentes ao aumento das admissões hospitalares, diminuição das listas de espera (waiting lists) para se obter cirurgia eletiva e aumento da cobertura vacinal. Uma primeira crítica a se fazer é que o governo assumiu que o crescimento das atividades de serviço seriam um indicador de que melhores resultados - outcomes - estariam sendo obtidos.

Mais ainda, os números apresentados não são comparados com estatísticas anteriores a 1990, o que nos deixa em dúvida se o crescimento de 1991-92 não seria parte de uma tendência de longo prazo ${ }^{6}$. Outro ponto a se ressaltar, é que a atividade hospitalar era tradicionalmente medida em função das altas efetuadas, e que o "relatório de sucessos" do governo instituiu uma nova medida de atividade número de vezes em que o paciente é examinado por diferentes especialidades. Acredita-se que este novo procedimento tenha aumentado artificialmente a atividade hospitalar ${ }^{6}$. Atitudes como esta fez com que a Royal Statistical Society ${ }^{7}$ sugerisse que serviços de estatísticas oficiais se desligassem do controle político por parte do governo. 
Quanto à diminuição das listas de espera para se obter cirurgia eletiva, 0 governo demonstrou a diminuição do número de pessoas esperando mais de 1 ano. Devido a popularidade do tema, o governo dispendeu um recurso especial de £35 milhões (atualmente a cotação da libra em relação ao dólar é £1 $=$ US $\$ 1.50$ ) com a recomendação explícita de ser usado na redução das listas até 31 de março (mês anterior às eleições parlamentares) ${ }^{8}$. Acredita-se portanto, que não haveria nenhuma relação direta desta diminuição com as recentes reformas e sim com o recurso extra investido. Evidências posteriores às eleiçōes mostraram uma elevação de $1.4 \%$ no número total de pessoas esperando cirurgias eletivas ${ }^{9}$.

Klein ${ }^{10}$, menos áspero com relação às reformas, comenta que "a transição vem sendo administrada com extraordinário sucesso e que os profetas da derrocada do NHS devem estar surpresos, porque o Sistema continuou a desempenhar as suas funçōes normalmente". Ele alerta entretanto, que os efeitos até agora detectados não são suficientes para avaliar a longo prazo, e que só assim se poderia criar uma linguagem comum que permitisse que as discordâncias pudessem ser discutidas de forma mais produtiva.

\section{A SEPARAÇÃO DO ATO DE COMPRAR SERVIÇOS DA AÇÃO DE PROVER SERVIÇOS (PURCHASER/PROVIDER SPLIT)}

Antes da reforma de abril de 1991, os distritos sanitários do Reino Unido (em torno de 193) eram responsáveis por prover serviços de saúde (ser proprietário 
de hospitais e outras unidades, além de empregar pessoal) e financiá-los (canalizar recursos para hospitais e outras unidades, baseado em orçamento anual e de acordo com a necessidade da população adstrita). Agora, muitos hospitais sairam do controle dos Distritos e adquiriram autonomia financeira administrativa - self-governing trusts vendendo serviços (através de contratos) para Distritos, GPs, e para o setor privado. Aqueles ainda sob controle distrital obtiveram maior autonomia e passam a negociar com os Distritos através de regime contratual análogo ao self-governing trusts ${ }^{3,11}$.

Harrison ${ }^{3}$ avalia as reformas do NHS como conseqüência de um processo longo e complexo desencadeado por pressōes políticas e econômicas que poderiam ser resumidas de uma forma simplificada na tão debatida "crise do welfare state". Por outro lado, Harrison apresenta um argumento que justificaria a racionalidade para a separação física e jurídica do ato de comprar/controlar serviços da ação de produzí-los. Nas instituições públicas monolíticas responsáveis pela prestação de serviços sociais pode-se discernir duas funçōes. O ato concreto de prover serviços e a atividade um pouco mais abstrata de identificar qual serviço deve ser provido. A localização destas duas funções na mesma agência não cria incentivos para eficiência e efetividade, uma vez que na identificação de necessidades, na provisão de serviços e na avaliação de resultados não há um conflito explícito, já que o mesmo grupo de profissionais está desenvolvendo as mesmas atividades. Em resumo, a separaçāo física e jurídica destas duas funções entre duas agências diferentes, produziriam espaço suficiente para a criação do conflito construtivo entre a provisão de serviços e 0 ato de avaliá-los de acordo com as necessidades previamente estabelecidas ${ }^{3}$. 
Combatida com veemência, esta separação entre provisão e compra de serviços é vista por muitos como uma tática do Partido Conservador para desestruturar - NHS, conduzindo inexoravelmente a um processo de privatizaçāo das unidades desintegradas $^{12,13}$. Entretanto algumas vozes inicialmente críticas à esta separação, como Partido Trabalhista (Labour) e a categoria médica (mais especificamente os profissionais com especializaçāo em saúde pública) começam a emitir sinais de que identificam alguns pontos positivos na proposta.

O Labour faz também a proposta da divisão das duas funções entre agências distintas, que se baseia em "comitês estratégicos" no nível distrital, responsáveis por determinar necessidades e prioridades locais, e "comitês operacionais" (hospitais e outras unidades) responsáveis por administrar a provisão de serviços. Entretanto, o mecanismo de financiamento proposto pelo Labour não cria mecanismos de competição entre as unidades provedoras de serviços como a atual proposta do Partido Conservador (ver Akerman, 1992 ${ }^{1}$ ).

Uma pesquisa com diretores de saúde pública distritais (médicos com especialização em saúde pública) demonstrou um entusiástico apoio ao purchaser-provider split ${ }^{14}$. Eles acreditam que a divisão de funções entre agências distintas dará ao Distrito maior possibilidade de definição mais precisa das necessidades da população e maior poder para comprar apenas serviços que contribuem para melhorar a saúde da população ("comprar saúde"). Seria o momento de sacrificar um pouco a independência dos profissionais de saúde pública em troca de maior influência nas decisões mais gerais ${ }^{10}$. 
Entretanto, Whitty e Jones ${ }^{16}$ discordam desta abordagem de "comprar saúde", uma vez que há evidências do restrito impacto da ação dos serviços sobre a saúde da população. Temem que esta idéia faça com que os "compradores de saúde" (gerentes administrativos) se sintam exercendo a função de saúde pública, e que os verdadeiros profissionais da área sejam vistos como supérfluos. Acrescentam que acham pouco provável a compra de serviços fora da esfera do setor saúde, uma vez que o NHS está carente de recursos financeiros e que usuários e prestadores de serviços fariam uma maior pressão para que os Distritos investissem em atendimento individual em detrimento de açōes que realmente promovessem saúde. Whitty e Jones acreditam que para os Distritos contrabalançarem o poder dos hospitais, eles terão que se fundir e muitos passarão a dar cobertura a populaçōes acima de 700000 habitantes o que prejudicará relações intersetoriais e efetiva definição das necessidades locais. Concluem dizendo que vêem um grande risco no envolvimento direto por parte dos profissionais de saúde pública na atividade de "compra de senviços".

Bhopal ${ }^{17}$ discorda desta conclusão e diz que os especialistas de saúde pública não podem abrir mão do seu papel em atividades de prestação de atendimento individual. Somente se envolvendo com as atividades ligadas ao cuidado é que eles poderão identificar necessidades da população, administrar a provisão e avaliar resultados. Só assim se poderá distinguir as açōes que produzem benefícios daquelas ineficientes ou mesmo deletérias.

Ham ${ }^{18}$ concorda com esta afirmação, e vê nos contratos de prestação de serviços firmados entre distritos e hospitais 0 instrumento adequado para a especificação da qualidade desejada em relação aos serviços contratados. De fato 
isto tem sido reforçado na medida que os distritos têm ameaçado, com a suspensão de contrato, aqueles hospitais que nāo cumprirem os padrōes estabelecidos. Instituem-se, portanto, com o purchaser-provider split mecanismos de prestação de contas (accountabilitty) inexistentes antes da reforma ${ }^{19}$.

\section{O REPASSE DE RECURSOS FINANCEIROS PARA AS CLÍNICAS DE GPS - GENERAL PRACTITIONER FUNDHOLDING}

Um dos principais componentes da reforma foi o estabelecimento de que consultórios de GPs com listas acima de 11000 pessoas poderão administrar o seu próprio orçamento, propiciando-Ihes, sem a intermediaçāo distrital, a compra direta de serviços farmacêuticos, laboratoriais, hospitalares e comunitários tanto do NHS quanto do setor privado. Acompanhando esta proposição, aparecem recomendações e incentivos de que qualquer economia obtida poderá ser revertida em benefício do consultório, seja na contrataçāo de pessoal auxiliar como na criação de novos serviços - fisioterapia ${ }^{20}$.

Aqueles favoráveis a esta proposição viam-na como uma oportunidade de fortalecimento dos GPs para advogar a causa de seus pacientes na obtenção dos serviços secundários necessários. Por outro lados os oponentes questionavam que a introdução de incentivos para se economizar recursos financeiros era perversa, e que poderia levar GPs a selecionar qual tipo de paciente que eles gostariam de ter em 
suas listas ${ }^{21}$. Temia-se ainda, que a introdução de mecanismos de mercado levaria a um aumento substancial dos custos administrativos 22 .

Newton et $\mathrm{al}^{21}$, acreditando que este debate carece de evidência empírica, realizaram um estudo onde através de entrevistas com GPs e pessoal auxiliar, descrevem a experiência de 10 consultórios no norte da Inglaterra após a implementação do esquema de fundholding. Os resultados confirmam a hipótese de que o esquema iria favorecer a obtenção de cuidados secundários, uma vez que os entrevistados foram quase unânimes em mencionar maior rapidez nos encaminhamentos e melhoria do cuidado hospitalar como um dos seus principais benefícios após a sua implantação. Quanto ao repasse financeiro, 7 consultórios conseguiram economizar algum recurso ( $1 \%$ a $4 \%$ do orçamento inicialmente planejado), enquanto os outros três gastaram de $1 \%$ a $3 \%$ a mais que o orçamento inicial. Não há especificação quanto ao gasto com atividades administrativas, mas os GPs reputaram o aumento do tempo e do esforço com atividades administrativas como a principal desvantagem até agora detectada. Quando questionados, os entrevistados refutaram com veemência as críticas dos opositores do fundholding de que considerações econômicas estariam influenciando suas decisōes clínicas. Entretanto, os autores propōem uma avaliação longitudinal mais rigorosa relacionada com esta questão.

Coulter ${ }^{20}$ levanta um outro problema relativo ao esquema do fundholding. Alguns consultórios estão estabelecendo firmas para que tenham possibilidade não apenas de comprar serviços, mas também de vendê-los. Acredita-se que isto faça com que haja indução de demanda por parte dos GPs de serviços que eles venham a prover. A experiência americana tem mostrado que isto leva a uma explosão de custos 
caso não haja uma regulação firme por parte do Estado ${ }^{20,22}$. Em outras palavras, as leis de mercado não são capazes de regular esta demanda induzida de serviços de saúde. Coulter adverte que fundholding deve ser visto como um experimento interessante, que deve ser acompanhado de perto, mas que a função de compra de serviços não deve ser transferida na sua totalidade para os GPs. Na realidade o esquema ainda está restrito a um pequeno número de consultórios bem administrados e que foram financiados generosamente. A experiência só poderá ser julgada a contento quando um maior número de consultórios se incorporar ao esquema e os resultados forem avaliados a longo prazo ${ }^{18}$.

\section{ASCONSEQÜÊNCIASDAREFORMAEMLONDRES:ORELATÓRIOTOMLINSON}

Os serviços de saúde em Londres custam, em média, 20\% a mais do que em qualquer outra parte da Inglaterra e o custo de atendimento de um caso num hospital de ensino londrino custa o dobro do mesmo tipo de atendimento num hospital geral ${ }^{23}$.

Em outubro de 1992 o governo divulgou um relatório ${ }^{4}$ que fazia um diagnóstico do sistema de saúde na cidade de Londres. Este diagnóstico foi acompanhado de uma terapêutica drástica que propôs o fechamento de 10 hospitais tradicionais da capital e fusão dos 9 atuais hospitais de ensino em apenas 4 hospitais-escola ${ }^{24,25}$. Especulava-se, anteriormente à implantação das reformas do 
NHS, que um dos seus objetivos seria o de interferir no poderoso serviços de saúde da capital. O relatório Tomlinson ${ }^{4}$ confirma estas suspeitas.

Estima-se que esta racionalização de recursos produza uma economia anual de £54 milhōes por ano e que este dinheiro seja investido em cuidados primários e em serviços comunitários ${ }^{4}$. Beardshaw ${ }^{26}$ concorda em princípio com Tomlinson, mas um programa de racionalização não pode apenas ficar restrito ao fechamento de hospitais. Ela sugere que o grupo de trabalho instituído pelo relatório Tomlinson deva equacionar a distribuição espacial dos serviços especializados de modo que eles reflitam as necessidades dos londrinos e não interesses institucionais e que deva estabelecer um sólido plano de assistência de urgência para a capital. Comenta-se que esta mudança de enfoque na prestação de serviços, com a redução de leitos hospitalares, exigirá dos hospitais de ensino inevitáveis mudanças na educação médica²7.

O Relatório Tomlinson é mais uma demonstraçāo da nova era por que passa o NHS. Caracterizado anteriormente por um processo de racionalização implícita, as condiçōes econômicas atuais impōem sobre o governo a tarefa de elaboração de procedimentos explícitos de racionalização no setor saúde ${ }^{3,28}$. Mas este processo não se dará de forma pacífica. $O$ londrino tem uma identidade muito forte com o "seu" hospital e tem feito fortes objeções a proposta de fechamento de várias unidades. 


\section{COMENTÁRIOS FINAIS}

Ham $^{18}$ aponta que o efeito mais importante da reforma até agora tem sido a mudança da relação de poder entre cuidado primário e hospitalar. A aliança dos Distritos com os GPs tem privilegiado o cuidado primário colocando os hospitais sob pressão. Os GPs vêm recebendo mais incentivos para a extensão de seus serviços enquanto os hospitais clamam por mais recursos, uma vez que muitos já esgotaram seu orçamento anual enquanto ainda faltam 3 meses para o fim do ano fiscal.

Aqueles consultórios de GPs com recurso disponível ainda podem encaminhar seus pacientes para cuidado hospitalar quando necessário, mas os GPs que não participam do esquema do fundholding terão em breve seus pacientes recusados quando o hospital de referência já tiver esgotado seu orçamento anual contratado. Acredita-se que não tardarão as manchetes jornalísticas a respeito de pacientes que não puderam ser atendidos por falta de recursos. Apesar de alguns benefícios obtidos com a reforma, o crônico problema da falta de recursos parece que ainda está por se resolver ${ }^{18}$.

E tudo indica que este problema há de permanecer. Enquanto escrevia este artigo o governo britânico lançava um pacote radical relacionado aos gastos públicos, e um jornal londrino publicava uma reportagem sobre este assunto, onde o setor saúde era mencionado como um dos "prejudicados", sob a manchete "Revisão total no welfare state" 29 . 
Contudo, $\operatorname{Sabin}^{30}$, mais realista, diz que todas as economias do primeiro mundo têm usado mecanismos de mercado como forma de controle. Um pouco mais otimista, talvez inspirado pela tradição do welfare state británico, Sabin pensa que o Reino Unido possa, através da mistura de valores sociais com mecanismos de mercado, criar um modelo de prestação de serviços para o próximo século.

\section{REFERÊNCIAS BIBLIOGRÁFICAS}

01-AKERMAN, M. As recentes mudanças do sistema de saúde britânico: alvoroço na saúde da corte. Saúde em Debate 34: 47-50, 1992.

02- DEPARTMENT OF HEALTH AND SOCIAL SECURITY. Working for patients. Londres, HMSO, 1989.

03- HARRISON, S. Working the markets: purchaser/provider separation in english health care. International Journal of Health Services, 21: 625-35, 1991.

04- TOMLINSON, B. Report of the inquiry into London's health service, medical education and research. Londres, HMSO, 1992.

05- NATIONAL HEALTH SERVICE MANAGEMENT EXECUTIVE. The NHS reforms: the first six months. Londres, HMSO, 1992.

06- RADICAL STATISTICS HEALTH GROUP. NHS reforms: the first six months - proof of progress or a statistical smokescreen? British Medical Journal, 304: 705-9, 1992.

07- ROYAL STATISTICAL SOCIETY. Official statistics: counting with confidence. The report of a working party on official statistics in the UK. London, Royal Statistical Society, 1990.

08- YATES, J. Lies, damned lies and waiting lists. British Medical Journal, 303: 802, 1991. 
09- THE GUARDIAN. NHS waiting list up after election, outubro de 1992.

10- KLEIN, R. NHS reforms: the first six months. British Medical Journal, 304: 199-200, $1992 a$.

11- FORSYTHE, M. Extracontractual referrals: the story so far. British Medical Journal, 303: 479-80, 1991.

12- KEEN, H. Why market forces cannot work. Medical Monitor. 1990.

13- REILLY, D. The fresh face of the NHS. British Medical Journal, 302: 1282, 1991.

14- FRANCOME, C. NHS reforms: the first six months: a survey of directors of public health. Enfield, Middlesex Polythecnic, Health Service Research Center, 1991.

15- GATHERER, A. Being executive. Public Health Physician, 2: 2, 1991.

16- WHITTY, P. \& JONES, I. Public health heresy: a challenge to the purchasing orthodoxy. British Medical Journal, 304: 1039-41, 1992.

17- BOPHAL, R.S. Public health medicine and purchasing health care. British Medical Journal, 306: 381-2, 1993.

18- HAM, C. How go the NHS reforms? British Medical Journal, 306: 77-8, 1993.

19- HOWELL, J.B.L. Re-examining the fundamental principles of the NHS. British Medical Journal, 304: 297-9, 1992.

20- COULTER, A. Fundholding general practices. British Medical Journal, 304: 397-8, 1992.

21- NEWTON, J.; FRASER, M.; ROBINSON, J. \& WAINRIGHT, D. Fundholding in Northern region: the first year. British Medical Journal, 306: 375-8, 1993.

22- NAVARRO, V. The relevance of the U.S. experience to the reforms of the British National Health Service: the case of general practitioner fund holding. International Journal of Health Senvices, 21: 381-7, 1991.

23- KING'S FUND. London Health Care 2010: changing the future of services in the capital, Londres, 1992. [mimeografado] 
24- DILLNER, L. Tomlinson report recommends London closures and merges. British Medical Journal, 305: 1045-6, 1992.

25- THE TOMLINSON report proposal in detail. The Independent Oct., 1992.

26- BEARDSHAW, V. Tomlinson reports on London. British Medical Journal, 305: 1039-40, 1992.

27- LOWRY, S. Trends in health care and their effects on medical education. British Medical Journal, 306: 255-8, 1993.

28- KLEIN, R. Warning signals from Oregon. British Medical Journal, 304: 1457-8, $1992 b$.

29- WELFARE state facing total review. The independent, Mar., 1993.

30- SABIN, J.E. "Mind the gap": reflections of an American health maintenace organisation doctor on the new NHS. British Medical Journal, 305: 514-6, 1992. 